\title{
ON WEIGHTED NORM INEQUALITIES FOR THE LUSIN AREA INTEGRAL
}

\author{
BY
}

\section{CARLOS SEGOVIA AND RICHARD L. WHEEDEN( $\left.{ }^{1}\right)$}

ABSTRACT. It is shown that the Lusin area integral for the unit circle is a bounded operator on any weighted $L^{p}$ space, $1<p<\infty$, on which the conjugate function is a bounded operator. Results are also proved for the case $0<p \leq 1$.

1. Introduction. The purpose of this paper is to derive several weighted norm inequalities for the Lusin area integral. Specifically, let $f(z), z=r e^{i \phi}$, be a function which is either harmonic or analytic in the disc $|z|<1$, and let

$$
S(f)(\theta)=\left(\iint_{\Gamma(\theta)}\left|(\nabla f)\left(r e^{i \phi}\right)\right|^{2} r d r d \phi\right)^{1 / 2},
$$

where $\Gamma(\theta)=\Gamma(\theta, \delta), 0<\delta<1$, is the open conical region bounded by the two tangents from $e^{i \theta}$ to $|z|=\delta$ and the more distant arc of $|z|=\delta$ between the points of contact. If $f$ is analytic and belongs to the Hardy space $H^{p}, 0<p<\infty$, then by [11]

$$
\left(\int_{0}^{2 \pi} S(f)^{p}(\theta) d \theta\right)^{1 / p} \leq c\left(\int_{0}^{2 \pi}|f(\theta)|^{p} d \theta\right)^{1 / p}
$$

where $f(\theta)$ denotes the boundary value of $f$ at $e^{i \theta}$ and $c$ is a constant independent of $f$. For a real-valued harmonic $f(z)$ which is the Poisson integral of $f(\theta)$, the same inequality holds for $1<p<\infty$ and there is of course a weak-type result when $p=1$.

For harmonic $f$ and $l<p<\infty$, we will be interested in deriving the inequality

$$
\left(\int_{0}^{2 \pi} S(f)^{p}(\theta) w(\theta) d \theta\right)^{1 / p} \leq c\left(\int_{0}^{2 \pi}|f(\theta)|^{p} w(\theta) d \theta\right)^{1 / p}
$$

for a large class of nonnegative periodic weight functions $w$. We will also give related results when $0<p \leq 1$. Some problems of this kind for very special

Received by the editors October 21, 1971.

AMS (MOS) subject classifications (1969). Primary 4430, 3067, 3035.

Key words and phrases. Lusin area integral, weighted norm inequalities, $H^{p}$ spaces, Poisson integrals.

(1) Part of this research was done while the second author was visiting the Facultad de Ciencias Exactas y Naturales, Buenos Aires. Moreover, it was partly supported by NSF GP-20132. 
weights $w$ have already been studied in [8] and [10].

In order to give a better description of the class of weight functions that we will consider, let us briefly recall the results proved in [7] and [13] for the conjugate function. Let $f(\theta), 0 \leq \theta<2 \pi$, be periodic and integrable over $(0,2 \pi)$, and let

$$
\tilde{f}(\theta)=\text { p.v. } \frac{1}{\pi} \int_{-\pi}^{\pi} \frac{f(\theta-\phi)}{2 \tan (\phi / 2)} d \phi
$$

be its conjugate function. In [7], H. Helson and G. Szegö proved the remarkable result that

$$
\left(\int_{0}^{2 \pi}|\tilde{f}(\theta)|^{2} w(\theta) d \theta\right)^{1 / 2} \leq c\left(\int_{0}^{2 \pi}|f(\theta)|^{2} w(\theta) d \theta\right)^{1 / 2}
$$

with $c$ independent of $f$ if and only if $w=e^{u+\widetilde{v}}$ where $u$ is a bounded function and $\tilde{v}$ is the conjugate (normalized as above) of a bounded function $v$ satisfying $\|v\|_{\infty}<\pi / 2$ (strict inequality). Whenever a weight function $w$ satisfies this condition we shall say that it has the Helson-Szegö form.

In [13] it is shown that $w$ has the Helson-Szegö form if and only if

$$
\left(\frac{1}{|I|} \int_{I} w(\theta) d \theta\right)\left(\frac{1}{|I|} \int_{I} w(\theta)^{-1} d \theta\right) \leq c,
$$

for every inteval $I$ which has length less than or equal to $2 \pi$ and center in $(0,2 \pi)$, with $c$ independent of $I$. Moreover, it is shown that a necessary and sufficient condition for the inequality

$$
\mathrm{C}_{p} \quad\left(\int_{0}^{2 \pi}|\tilde{f}(\theta)|^{p} w(\theta) d \theta\right)^{1 / p} \leq c\left(\int_{0}^{2 \pi}|f(\theta)|^{p} w(\theta) d \theta\right)^{1 / p}
$$

for a given $p, 1<p<\infty$, is that $w$ satisfy the condition

$$
A_{p} \quad\left(\frac{1}{|I|} \int_{I} w(\theta) d \theta\right)\left(\frac{1}{|I|} \int_{I} w(\theta)^{-1 /(p-1)} d \theta\right)^{p-1} \leq c
$$

with $c$ independent of $I$, for intervals $I$ of the kind described above.

A simple application of Hölder's inequality shows that if $w$ satisfies $A_{p}$ for a given $p$ then it also satisfies $\mathrm{A}_{q}$ for any $q$ with $p \leq q<\infty$. In particular, if $w$ satisfies $A_{p}\left(C_{p}\right)$ for some $p<2$ then it satisfies $A_{2}\left(C_{2}\right)$, and so has the Helson-Szegö form.

It is simple to check that $w$ satisfies $A_{p}$ for some $p, 1<p<\infty$, if and only if $w^{-1 /(p-1)}$ satisfies $A_{p^{\prime}}, 1 / p+1 / p^{\prime}=1$. Thus if $w$ satisfies $A_{p}\left(C_{p}\right)$ for some $p>2$, then since $p^{\prime}<2, w^{-1 /(p-1)}$ has the Helson-Szegö form.

A certain weak-type result $C_{1}$ for $p=1$ is also derived in [13], the necessary and sufficient condition then being 
$A_{1}$

$$
w^{*}(\theta) \leq c w(\theta)
$$

where

$$
w^{*}(\theta)=\operatorname{Sup}_{I \ni \theta ;|I| \leq 2 \pi} \frac{1}{|I|} \int_{I} w(\phi) d \phi
$$

is the Hardy-Littlewood maximal function of $w$.

In the case $p>1$, our main result is that any weight which satisfies $C_{p}$ also satisfies (1.1). In fact we shall prove the following theorem.

Theorem 1. Let $1<p<\infty$. If $w$ satisfies $\mathrm{C}_{p}$ and $f$ is a barmonic function which satisfies

$$
\operatorname{Sup}_{0<r<1}\left(\int_{0}^{2 \pi}\left|f\left(r e^{i \theta}\right)\right|^{p} w(\theta) d \theta\right)^{1 / p}=M<\infty
$$

then

$$
\left(\int_{0}^{2 \pi} S(f)^{p}(\theta) w(\theta) d \theta\right)^{1 / p} \leq c M
$$

with $c$ independent of $f$.

As we shall show in Lemma 6 below, hypothesis (1.2) for a $w$ satisfying $A_{p}$ actually amounts to assuming that $f\left(r e^{i \theta}\right)$ is the Poisson integral of a function $f(\theta)$ satisfying

$$
c_{1} M \leq\left(\int_{0}^{2 \pi}|f(\theta)|^{p} w(\theta) d \theta\right)^{1 / p} \leq c_{2} M .
$$

We prove the case $p \geq 2$ of Theorem 1 in $\$ 3$. In $\$ 4$, we prove the case 1 $<p<2$ as well as state and derive several related results and corollaries for $0<p \leq 1$. $\$ 2$ contains various known facts and lemmas that we will need in the later sections.

This paper is intended as a step in finding weighted versions of LittlewoodPaley theorems. It leaves open several interesting problems, such as nonperiodic versions, extensions to higher dimensions and the validity of an inequality opposite to that given in Theorem 1. These questions will be treated in a sequel to this paper. We are indebted to Professor B. Muckenhoupt and Professors C. Fefferman and E. M. Stein for making preprints of their papers [12] and [4] available to us.

2. Preliminaries. We begin by observing that if $w$ satisfies condition $A_{p}$, $1<p<\infty$, then

$$
\frac{1}{|I|} \int_{I} w(\theta) d \theta \approx\left(\frac{1}{|I|} \int_{I} w(\theta)^{-1 /(p-1)} d \theta\right)^{-(p-1)}
$$


by which we mean that there exist positive constants $c_{1}$ and $c_{2}$ such that

$$
c_{1} \leq\left(\frac{1}{|I|} \int_{I} w(\theta) d \theta\right)\left(\frac{1}{|I|} \int_{I} w(\theta)^{-1 /(p-1)} d \theta\right)^{p-1} \leq c_{2}
$$

for all relevant intervals $I$. The right-hand inequality is just $A_{p}$; and the lefthand inequality with $c_{1}=1$ follows by applying Hölder's inequality to $1=$ $|I|^{-1} \int_{I} w(\theta)^{1 / p} w(\theta)^{-1 / p} d \theta$ with exponents $p$ and $p^{\prime}, 1 / p+1 / p^{\prime}=1$.

Suppose now that $w$ has the special form $w(\theta)=e^{\tilde{v}(\theta)}$ where $v(\theta)$ is a bounded function. With such a function $w$ we shall associate the analytic function $W(z)=e^{\tilde{\nu}(z)-i v(z)}$, where $v(z)$ and $\tilde{v}(z)$ denote the Poisson and conjugate Poisson integrals of $v(\theta)$. (See also [7].) Thus

$$
|W(z)|=e^{\tilde{\nu(z)}} \text { has boundary values } e^{\widetilde{\nu}(\theta)}=w(\theta) \text {. }
$$

If, in addition, $\|v\|_{\infty}<\pi / 2$ (strict inequality) then $w$ is integrable over $(0$, $2 \pi$ ). (In fact, by [14, p. 254], $w$ is integrable to a power $\left.p_{1}>1.\right)$ In this case, let $w(z)$ denote the Poisson integral of $w$. Since $\widetilde{v}(z)$ is the Poisson integral of $\log w(\theta)$, it follows easily from Jensen's inequality for convex functions that $|W(z)| \leq w(z)$. In particular, $W \in H^{1}$. Since $|v(z)| \leq c<\pi / 2,|W(z)| \approx \operatorname{Re}[W(z)]$. (See [6] and [7, p. 131].) Since the harmonic function $\operatorname{Re}[W(z)]$ is the Poisson integral of its boundary values, and the se boundary values are $e^{\widetilde{\nu}(\theta)} \cos v(\theta)=$ $w(\theta) \cos \nu(\theta) \approx w(\theta)$, we have

$$
|W(z)| \approx w(z) \text { if } w(\theta)=e^{\widetilde{v}(\theta)} \text { with }\|v\|_{\infty}<\pi / 2
$$

In particular, if we denote $w(\theta)^{-1}=e^{-\widetilde{v}(\theta)}$ by $w_{1}(\theta)$ and observe that $w_{1}$ has the same form as $w$, we see that its Poisson integral $w_{1}(z) \approx\left|W(z)^{-1}\right|$. Thus,

$$
w(z) w_{1}(z) \approx 1 \quad \text { if } w(\theta)=e^{\widetilde{v}(\theta)} \text { with }\|v\|_{\infty}<\pi / 2
$$

Lemma 1. If $w(\theta)=e^{\widetilde{\nu}(\theta)}$ with $\|v\|_{\infty}<\pi / 2$ then $w$ satisfies $\mathrm{A}_{2}$ and

$$
w\left(r e^{i \theta}\right) \approx \frac{1}{1-\dot{r}} \int_{|\theta-\phi|<1-r} w(\phi) d \phi .
$$

Simple estimates on the Poisson kernel $P(r, \theta)$ show that $P(r, \theta) \approx$ $1 /(1-r)$ for $|\theta|<1-r$. Thus for any Poisson integral $g\left(r e^{i \theta}\right)=$ $\int_{-\pi}^{\pi} g(\theta-\phi) P(r, \phi) d \phi$ with $g \geq C$, we have

$$
g\left(r e^{i \theta}\right) \geq \frac{c}{1-r} \int_{|\phi|<1-r} g(\theta-\phi) d \phi
$$

Therefore, by (2.4),

$$
c_{1} \geq w\left(r e^{i \theta}\right) w_{1}\left(r e^{i \theta}\right) \geq c w\left(r e^{i \theta}\right)\left(\frac{1}{1-r} \int_{|\theta-\phi|<1-r} w(\phi)^{-1} d \phi\right),
$$


which in turn implies that

$$
c_{1} \geq c\left(\frac{1}{1-r} \int_{|\theta-\phi|<1-r} w(\phi) d \phi\right)\left(\frac{1}{1-r} \int_{|\theta-\phi|<1-r} w(\phi)^{-1} d \phi\right) .
$$

This inequality shows that $w$ satisfies $A_{2}$ for all intervals $I$ of length less than 1. But since $w$ and $w^{-1}$ are integrable, the condition for $1 \leq|I| \leq 2 \pi$ is immediate. Thus $w$ satisfies $A_{2}$. But then the former inequality together with (2.1) for $p=2$ gives

$$
w\left(r e^{i \theta}\right) \leq \frac{c}{1-r} \int_{|\theta-\phi|<1-r} w(\phi) d \phi .
$$

The following two important properties of weights $w$ satisfying $A_{p}$ are proved in [12].

Lemma 2. Let $f^{*}(\theta)=\operatorname{Sup}_{I \ni \theta,|I| \leq 2 \pi}|I|^{-1} \int_{I}|f(\phi)| d \phi$ be the Hardy-Littlewood maximal function of $f$. Then a necessary and sufficient condition for the inequality

$$
\left(\int_{0}^{2 \pi} f^{*}(\theta)^{p} w(\theta) d \theta\right)^{1 / p} \leq c\left(\int_{0}^{2 \pi}|f(\theta)|^{p} w(\theta) d \theta\right)^{1 / p}
$$

for some $p, 1<p<\infty$, is that $w$ satisfy $A_{p}$.

A weak-type version of Lemma 2 for $p=1$ was obtained earlier by C. Fefferman and E. M. Stein [5].

It follows easily from Hölder's inequality that a weight $w$ which satisfies $A_{p}$ also satisfies $A_{q}$ for all $q \geq p$. The next lemma states essentially that $w$ also satisfies $A_{p-\epsilon}$ for sufficiently small $\epsilon>0$.

Lemma 3. If $w$ satisfies $A_{p}$ for some $p, 1<p<\infty$, there exists $p_{1}>1$ such that $w^{p_{1}}$ satisfies $\mathrm{A}_{p}$ and

$$
\left(\frac{1}{|I|} \int_{I} w(\theta)^{p_{1}} d \theta\right)^{1 / p_{1}} \leq \frac{c}{|I|} \int_{I} w(\theta) d \theta
$$

for all $I$

The next lemma states some useful known relations between Poisson integrals and Hardy-Littlewood maximal functions.

Lemma 4. Let $g(z)$ be the Poisson integral of a nonnegative function $g$ and let $\Gamma\left(r e^{i \theta}\right)$ be the conical region bounded by the tangents from re $e^{i \theta}$ to $|z|=\delta$ and the more distant arc of $|z|=\delta$ between the points of contact. Then

$$
\operatorname{Sup}_{\Gamma\left(r e^{i \theta}\right)} g(z) \geq c \operatorname{Sup}_{1-r \leq \epsilon \leq \pi} \frac{1}{\epsilon} \int_{|\phi|<\epsilon} g(\theta+\phi) d \phi .
$$

Moreover, there is a constant $\gamma$ which depends on $\delta$ such that 


$$
\operatorname{Sup}_{\Gamma\left(r e^{i \theta}\right)} g(z) \leq c \quad \operatorname{Sup}_{\gamma(1-r) \leq \epsilon \leq \pi} \frac{1}{\epsilon} \int_{|\phi|<\epsilon} g(\theta+\phi) d \phi .
$$

Recalling that $(1-r)|\nabla P(r, \theta)| \leq c P(r, \theta)$ for $|\theta| \leq \pi$ we obtain as a special corollary of Lemma 4 that $(1-r)\left|\nabla g\left(r e^{i \theta}\right)\right|$ is majorized by a constant times the Hardy-Littlewood maximal function $g^{*}(\theta)$ of $g$.

The following lemma, which is due to L. Carleson [3], and some of its variants play an important role in what follows.

Lemma 5. Let $\mu(z)$ be a nonnegative measure in $|z|<1$ which satisfies $\mu(S) \leq c l$ for all sets $S$ of the form $S=\left\{r e^{i \theta}: r \geq 1-l, \theta_{0} \leq \theta \leq \theta_{0}+l\right\}, 0<l \leq 1$. Then there is a constant $A$ depending only on $p$ so that

$$
\left(\iint_{|z|<1}|G(z)|^{p} d \mu(z)\right)^{1 / p} \leq A c\|G\|_{p}
$$

for all $G \in H^{p}, p>0$.

This lemma has been generalized and given simpler proofs in [9] and [5]. We will need the lemma as stated as well as in an analogous form for special subdomains of $|z|<1$. The simple proof given in [5] is especially helpful in obtaining this.

Lemma 6. Suppose that $w$ satisfies $A_{p}$ for some $p, 1<p<\infty$, and let $f\left(r e^{i \theta}\right)$ be barmonic in $|z|<1$. Then

$$
\operatorname{Sup}_{0<r<1}\left(\int_{0}^{2 \pi}\left|f\left(r e^{i \theta}\right)\right|^{p} w(\theta) d \theta\right)^{1 / p}=M<\infty
$$

if and only if $f\left(r e^{i \theta}\right)$ is the Poisson integral of a function $f(\theta)$ satisfying

$$
\left(\int_{0}^{2 \pi}|f(\theta)|^{p} w(\theta) d \theta\right)^{1 / p}<\infty .
$$

Moreover, $\left(\int_{0}^{2 \pi}|f(\theta)|^{p} w(\theta) d \theta\right)^{1 / p} \approx M$.

First we note that any function $g(\theta)$ for which $\int_{0}^{2 \pi}|g(\theta)|^{p} u(\theta) d \theta<\infty$ necessarily belongs to $L^{p_{1}}$ for some $p_{1}>1$. In fact, choosing $1<p_{1}<p$, we have from Hölder's inequality that

$$
\begin{aligned}
\int_{0}^{2 \pi}|g(\theta)|^{p} d \theta & =\int_{0}^{2 \pi}|g(\theta)|^{p} 1^{p} w(\theta)^{p_{1} / p} \cdot w(\theta)^{-p_{1} / p} d \theta \\
& \leq\left(\int_{0}^{2 \pi}|g(\theta)|^{p} w(\theta) d \theta\right)^{p_{1} / p}\left(\int_{0}^{2 \pi} w(\theta)^{-\left(p / p_{1}-1\right)^{-1}} d \theta\right)^{1-p_{1} / p} .
\end{aligned}
$$

But $\left(p / p_{1}-1\right)^{-1}$ converges to $1 /(p-1)$ from above as $p_{1} \rightarrow 1$ and $w^{1+\epsilon}$ satisfies $A_{p}$ (Lemma 3$)$. Therefore the second integral on the right is finite for $p_{1}$ sufficiently close to 1 . 
Thus if $f$ satisfies (2.6), then

$$
\operatorname{Sup}_{0<r<1}\left(\int_{0}^{2 \pi}\left|f\left(r e^{i \theta}\right)\right|^{p} 1 d \theta\right)^{1 / p_{1}}<\infty
$$

for some $p_{1}>1$. Since $f$ is harmonic it must then be the Poisson integral of a function $f(\theta)$. Hence $f\left(r e^{i \theta}\right)$ converges to $f(\theta)$ almost everywhere as $r \rightarrow 1$, and it follows from (2.6) and Fatou's lemma that

$$
\left(\int_{0}^{2 \pi}|f(\theta)|^{p} w(\theta) d \theta\right)^{1 / p} \leq M
$$

Conversely, if $f\left(r e^{i \theta}\right)$ is the Poisson integral of a function $f(\theta)$ satisfying (2.7) then

$$
\left(\int_{0}^{2 \pi}\left|f\left(r e^{i \theta}\right)\right|^{p} w(\theta) d \theta\right)^{1 / p} \leq c\left(\int_{0}^{2 \pi}\left|f^{*}(\theta)\right|^{p} w(\theta) \mid d \theta\right)^{1 / p}
$$

where $f^{*}$ is the Hardy-Littlewood maximal function of $f(\theta)$. (2.6) now follows from Lemma 2.

3. The case $2 \leq p<\infty$. In this section we will prove Theorem 1 for $p \geq 2$. Thus let $w(\theta)$ satisfy condition $C_{p}, p \geq 2$. As was noted in the introduction, this is the same as supposing that $w$ satisfies $A_{p}$ and implies that $w^{-1 /(p-1)}$ is of Helson-Szegö form. For such $w$ and any analytic function $F$ satisfying

$$
\operatorname{Sup}_{0<r<1}\left(\int_{0}^{2 \pi}\left|F\left(r e^{i \theta}\right)\right|^{p} w(\theta) d \theta\right)^{1 / p}<\infty
$$

we will show that

$$
\left(\int_{0}^{2 \pi} S(F)^{p}(\theta) w(\theta) d \theta\right)^{1 / p} \leq c\left(\int_{0}^{2 \pi}\left|F\left(e^{i \theta}\right)\right|^{p} w(\theta) d \theta\right)^{1 / p} .
$$

Here $F\left(e^{i \theta}\right)=\lim _{r \rightarrow 1} F\left(r e^{i \theta}\right)$ exists almost everywhere since $F \in H^{p} 1, p_{1}>1$ (see the proof of Lemma 6).

This fact together with Lemma 6 and the hypothesis that $w$ satisfies $C_{p}$ will prove The orem 1 for $p \geq 2$. Of course, if $f$ is the conjugate of a harmonic function $f$ and $F$ is the analytic function $F=f+i f$, then $S(F)(\theta)=2 S(f)(\theta)$.

Since $w^{-1 /(p-1)}$ is of Helson-Szegö form, we may write $w=e^{u+\widetilde{v}}$ where $u$ and $v$ are bounded and $\|v\|_{\infty}<(p-1)_{\pi} / 2$. Since $e^{u}$ is bounded above and also below away from zero, we may assume that $u=0$-that is, that $w=e^{\tilde{v}}$. If $W(z)=e^{\tilde{\nu}(z)-i v(z)}$ is the analytic function associated with $w$ then $W(z)^{-1(p-1)}$ is the one associated with $w^{-1 /(p-1)}$. Since $w \geq 0$, Harnack's principle applied to (2.5) for $w^{-1 /(p-1)}$ and its Poisson integral, $w_{1}\left(r e^{i \theta}\right)$, gives 


$$
w_{1}\left(r e^{i \theta}\right) \approx \frac{1}{1-r} \int_{\gamma|\theta-\phi|<1-r} w(\phi)^{-1 /(p-1)} d \phi
$$

The constants giving this equivalence of course depend on $\gamma$. Combining this with (2.3) we obtain

$$
\left|W\left(r e^{i \theta}\right)\right|^{-1 /(p-1)} \approx \frac{1}{1-r} \int_{\gamma|\theta-\phi|<1-r} w(\phi)^{-1 /(p-1)} d \phi .
$$

Hence by (2.1),

$$
\left|W\left(r e^{i \theta}\right)\right| \approx \frac{1}{1-r} \int_{\gamma|\theta-\phi|<1-r} w(\phi) d \phi .
$$

Since $p / 2 \geq 1,(3.2)$ will follow from proving that there exists a constant $c$ such that

$$
\int_{0}^{2 \pi} S(F)(\theta)^{2} w(\theta)^{2 / p} b(\theta) d \theta \leq c\left(\int_{0}^{2 \pi}\left|F\left(e^{i \theta}\right)\right|^{p} w(\theta) d \theta\right)^{2 / p}
$$

for every nonnegative $b \in L^{(p / 2)^{\prime}}$ with $\|b\|_{(p / 2)^{\prime}} \leq 1$. When $p=2$ we will always assume, as we may, that $b \equiv 1$.

Substituting

$$
S(F)(\theta)^{2}=\iint_{\Gamma(\theta)}\left|F^{\prime}\left(r e^{i \phi}\right)\right|^{2} r d r d \phi
$$

and then changing the order of integration, we find it is enough to show that

$$
\iint_{|z|<1} K(r, \phi)\left|F^{\prime}\left(r e^{i \phi}\right)\right|^{2} r d r d \phi \leq c\left(\int_{0}^{2 \pi}\left|F\left(e^{i \phi}\right)\right|^{p} w(\phi) d \phi\right)^{2 / p}
$$

where

$$
K(r, \phi)=\int_{\gamma|\theta-\phi|<1-r} w^{2 / p}(\theta) b(\theta) d \theta,
$$

$\gamma$ being a constant depending on $\delta$. Here we have used the fact that there is a constant $c$ so that

$$
\left|F^{\prime}(z)\right| \leq c\left(\int_{0}^{2 \pi}\left|F\left(e^{i \phi}\right)\right|^{p} w(\phi) d \phi\right)^{1 / p} \quad \text { for all }|z|<\delta .
$$

In fact, it follows easily from Cauchy's integral formula for the derivative of an analytic function that $\left|F^{\prime}(z)\right| \leq c \int_{0}^{2 \pi}\left|F\left(e^{i \phi}\right)\right| d \phi$ for $|z|<\delta$. Then by Hölder's inequality and the fact that $w^{-p^{1} / p}=w^{-1 /(p-1)}$ is integrable,

$$
\left|F^{\prime}(z)\right| \leq c\left(\int_{0}^{2 \pi}\left|F\left(e^{i \phi}\right)\right|^{p} w(\phi) d \phi\right)^{1 / p} \text { for }|z|<\delta
$$

as claimed. 
Let $I=I(r, \phi)=\{\theta: y|\theta-\phi|<1-r\}$ and let $s=(p / 2)(1+\epsilon)$ for a small $\epsilon>0$ to be chosen. By Hölder's inequality,

$$
K(r, \phi) \leq|I|\left(\frac{1}{|I|} \int_{I} w^{2 s / p}(\theta) d \theta\right)^{1 / s}\left(\frac{1}{|I|} \int_{I} b^{s^{\prime}}(\theta) d \theta\right)^{1 / s^{\prime}} .
$$

If we now choose $\epsilon$ sufficiently small and apply Lemma 3, we find that

$$
K(r, \phi) \leq c|I|\left(\frac{1}{|I|} \int_{I} w(\theta) d \theta\right)^{2 / p}\left(\frac{1}{|I|} \int_{I} b^{s^{\prime}}(\theta) d \theta\right)^{1 / s^{\prime}} .
$$

By (3.3) and the definition of $I$,

$$
K(r, \phi) \leq c\left(1-r^{2}\right)\left|W\left(r e^{i \phi}\right)\right|^{2 / p} g\left(r e^{i \phi}\right)^{1 / s^{\prime}},
$$

where $g$ denotes the Poisson integral of $b^{s^{\prime}}$. Of course, $s^{\prime}<(p / 2)^{\prime}$, and therefore $b^{s^{\prime}}$ is integrable to a power strictly larger than 1 . The expression on the left in (3.4) is therefore at most a constant times

$$
\iint_{|z|<1}\left(1-r^{2}\right) g\left(r e^{i \phi}\right)^{1 / s^{\prime}}\left|W\left(r e^{i \phi}\right)^{1 / p} F^{\prime}\left(r e^{i \phi}\right)\right|^{2} r d r d \phi .
$$

Since $\left(W^{1 / p} F\right)^{\prime}=W^{1 / p} F^{\prime}+(1 / p) W^{1 / p-1} W^{\prime} F$, it is enough to show the bound in question for both

$$
\iint_{|z|<1}\left(1-r^{2}\right) g\left(r e^{i \phi}\right)^{1 / s^{\prime}}\left|\left\{W\left(r e^{i \phi}\right)^{1 / p} F\left(r e^{i \phi}\right)\right\}^{\prime}\right|^{2} r d r d \phi
$$

and

$$
\iint_{|z|<1}\left(1-r^{2}\right) g\left(r e^{i \phi}\right)^{1 / s^{\prime}}\left|W\left(r e^{i \phi}\right)^{1 / p-1} W^{\prime}\left(r e^{i \phi}\right) F\left(r e^{i \phi}\right)\right|^{2} r d r d \phi .
$$

We recall that $g=b=1$ when $p=2$.

We will estimate (3.5) first. As we easily obtain from changing the order of integration, (3.5) is majorized by a constant times

$$
\int_{0}^{2 \pi}\left[\iint_{\Gamma(\theta)} g\left(r e^{i \phi}\right)^{1 / s^{\prime}}\left|\left\{W\left(r e^{i \phi}\right) F\left(r e^{i \phi}\right)\right\}^{\prime}\right|^{2} r d r d \phi\right] d \theta .
$$

Denoting $g^{*}(\theta)=\operatorname{Sup}_{\Gamma(\theta)} g\left(r e^{i \phi}\right)$, we see this is at most

$$
\begin{gathered}
\int_{0}^{2 \pi} g^{*}(\theta)^{1 / s^{\prime}}\left[\iint_{\Gamma(\theta)}\left|\left\{W^{1 / p}\left(r e^{i \phi}\right) F\left(r e^{i \phi}\right)\right\}^{\prime}\right|^{2} r d r d \phi\right] d \theta \\
=\int_{0}^{2 \pi} g^{*}(\theta)^{1 / s^{\prime}} s\left(W^{1 / p} F\right)(\theta)^{2} d \theta . .
\end{gathered}
$$


To this expression we apply Hölder's inequality with exponents $(p / 2)^{\prime}$ and $p / 2$, obtaining the bound $\left\|g^{*}\right\|_{(p / 2)^{\prime}\left(1 / s^{\prime}\right)}^{1 / s^{\prime}}\left\|S\left(W^{1 / p} F\right)\right\|_{p}^{2}$.

Since $g$ is the Poisson integral of $b^{s^{\prime}}$ and $(p / 2)^{\prime}\left(1 / s^{\prime}\right)>1$,

$$
\left\|g^{*}\right\|_{(p / 2)^{\prime}\left(1 / s^{\prime}\right)}^{1 / s^{\prime}} \leq c\|b\|_{(p / 2)^{\prime}} \leq c .
$$

Moreover, the analytic function $W^{1 / p} F \in H^{p}$-for by $[14, \mathrm{p} .273]$, and by (2.2)

$$
\int_{0}^{2 \pi}\left|W\left(r e^{i \theta}\right)^{1 / p} F\left(r e^{i \theta}\right)\right|^{p} d \theta \leq \int_{0}^{2 \pi} w(\theta)\left|F\left(e^{i \theta}\right)\right|^{p} d \theta<\infty .
$$

Hence, applying the unweighted version of the area integral theorem, we get

$$
\left\|S\left(W^{1 / p} F\right)\right\|_{p}^{2} \leq c\left\|W\left(e^{i \theta}\right)^{1 / p} F\left(e^{i \theta}\right)\right\|_{p}^{2}=c\left(\int_{0}^{2 \pi}\left|F\left(e^{i \theta}\right)\right|^{p} w(\theta) d \theta\right)^{2 / p} .
$$

This completes the estimate for (3.5).

To estimate (3.6), it will be convenient to let $G$ denote the analytic function $g+i \tilde{g}$. Since $b \geq 0$ and is not identically zero, $g\left(r e^{i \phi}\right)>0$, so that powers of $G$ are well defined and analytic. Moreover, $g^{1 / s^{\prime}} \leq|G|^{1 / s^{\prime}}=\left|G^{1 / s^{\prime}}\right|$ and therefore (3.6) is at most

$$
\iint_{|z|<1}\left(1-r^{2}\right)\left|W^{1 / p-1} W^{\prime} G^{1 / 2 s^{\prime}} F\right|^{2} r d r d \phi .
$$

Since $\left|W^{\prime}\right|=|W|\left|(\tilde{v}-i v)^{\prime}\right|=|W||\nabla \nu|$ by the Cauchy-Riemann equations, we may rewrite the expression above as

$$
\iint_{|z|<1}\left|W^{1 / p} G^{1 / 2 s^{\prime}} F\right|^{2}\left(1-r^{2}\right)|\nabla v|^{2} r d r d \phi .
$$

We claim that the measure $d \mu=\left(1-r^{2}\right)|\nabla v|^{2} r d r d \phi$ satisfies the condition of Lemma 5. Thus by applying that lemma to the analytic function $W^{1 / p} G^{1 / 2 s^{\prime}} F$, we see the last integral is at most a constant times

$$
\int_{0}^{2 \pi}\left|G\left(e^{i \theta}\right)\right|^{1 / s^{\prime}}\left|F\left(e^{i \theta}\right)\right|^{2} w(\theta)^{2 / p} d \theta
$$

By Hölder's inequality, this is at most

$$
\|G\|_{(p / 2)^{\prime}\left(1 / s^{\prime}\right)}^{1 / s^{\prime}}\left(\int_{0}^{2 \pi}\left|F\left(e^{i \theta}\right)\right|^{p} w(\theta) d \theta\right)^{2 / p}
$$

When $p=2, G=1$ and this is the desired estimate of (3.6). When $p>2$ then $1<(p / 2)^{\prime}\left(1 / s^{\prime}\right)<\infty$, and since the real part of $G$ on $|z|=1$ is $b^{s^{\prime}}$, we obtain from well-known norm inequalities for the conjugate function that $\|G\|_{(p / 2)^{\prime}\left(1 / s^{\prime}\right)}^{1 / s^{\prime}}$ $\leq c\|b\|_{(p / 2)^{\prime}} \leq c$. This will complete the estimation of (3.6). 
To show that $d \mu\left(r e^{i \phi}\right)=\left(1-r^{2}\right)\left|\nabla \nu\left(r e^{i \phi}\right)\right|^{2} r d r d \phi$ satisfies the condition of Lemma 5, let $S$ be a region of the kind described there. Then

$$
\mu(S)=\iint_{S}\left(1-r^{2}\right)\left|\nabla v\left(r e^{i \phi}\right)\right|^{2} r d r d \phi=\frac{1}{2} \iint_{S}\left(1-r^{2}\right) \Delta v^{2}\left(r e^{i \phi}\right) r d r d \phi .
$$

We want to apply Green's theorem to this expression. Thus let $S_{k}, k=1,2, \ldots$, be an increasing sequence of approximating domains for $S$-that is, the $S_{k} \subset S$, have closures contained in $|z|<1$ and have smooth boundaries $\partial S_{k}$ whose lengths are uniformly bounded by a multiple of the length of $\partial S$. Since $\Delta(1-r)^{2}$ $=-4$ is negative,

$$
\begin{aligned}
& \iint_{S_{k}}\left(1-r^{2}\right) \Delta v^{2}\left(r e^{i \phi}\right) r d r d \phi \\
& \quad \leq \int_{\partial S_{k}}\left(1-r^{2}\right)\left|\frac{\partial}{\partial \eta_{k}} v^{2}\left(r e^{i \phi}\right)\right| d \sigma_{k}+\int_{\partial S_{k}} v^{2}\left(r e^{i \phi}\right)\left|\frac{\partial}{\partial \eta_{k}}\left(1-r^{2}\right)\right| d \sigma_{k},
\end{aligned}
$$

where $\partial / \partial \eta_{k}$ denotes differentiation along the normal to $\partial S_{k}$ and $d \sigma_{k}$ denotes the element of arc length along $\partial S_{k}$. The second integral on the right is clearly bounded by a constant times $\|v\|_{\infty}^{2}$ (length of $\partial S_{k}$ ) $\leq c\|v\|_{\infty}^{2} l$. Moreover, since $\left(1-r^{2}\right)\left|\nabla v\left(r e^{i \phi}\right)\right| \leq c v^{*}(\phi)$, where $v^{*}$ is the Hardy-Littlewood maximal function of $v$, and $\left\|v^{*}\right\|_{\infty} \leq\|v\|_{\infty}$, the first integral on the right is

$$
\begin{aligned}
2 \int_{\partial S_{k}}\left|v\left(r e^{i \phi}\right)\right|\left(1-r^{2}\right)\left|\frac{\partial}{\partial \eta_{k}} v\left(r e^{i \phi}\right)\right| d \sigma_{k} \\
\left.\leq c\|v\|_{\infty}^{2} \text { (length of } \partial S_{k}\right) \leq c\|v\|_{\infty}^{2} l .
\end{aligned}
$$

4. The case $0<p<2$. In this section we will complete the proof of Theorem 1 in the remaining case $1<p<2$ and prove results for harmonic (analytic) functions for $0<p<2$. The basic outline of the proof we will use is that given by C. Fefferman and E. M. Stein [4] in generalizing results of D. L. Burkholder, R. F. Gundy and M. L. Silverstein [1].

To fix the notation, let $f\left(r e^{i \phi}\right)$ be harmonic in $|z|<1$ and let

$$
S(f)(\theta)=\left(\iint_{\Gamma(\theta)}\left|\nabla f\left(r e^{i \phi}\right)\right|^{2} r d r d \phi\right)^{1 / 2},
$$

where $\Gamma(\theta)=\Gamma(\theta, \delta)$. For a $\delta_{1}>\delta$, let $\Gamma_{1}(\theta)=\Gamma\left(\theta, \delta_{1}\right)$ so that $\Gamma(\theta) \subset \Gamma_{1}(\theta)$. Now let

$$
f^{*}(\theta)=\operatorname{Sup}_{\Gamma_{1}(\theta)}\left|f\left(r e^{i \theta}\right)\right|
$$


The main result of this section is then the following theorem.

Theorem 2. Let $w(\theta)$ satisfy $A_{2}$ and let $f\left(r e^{i \phi}\right)$ be barmonic in $|z|<1$. Then for $\alpha>0$,

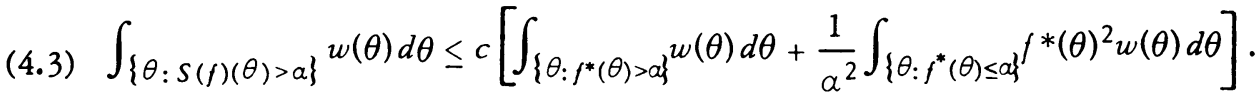

The constant $c$ is independent of $f$ and $a$.

The same result for $w=1$ is proved in [4]. The proof of Theorem 2 constitutes the main part of this section. As a corollary we obtain

Theorem 3. Let $f$ be a barmonic function and let $w$ satisfy $\mathrm{A}_{2}$. Then for $0<p<2$,

$$
\left(\int_{0}^{2 \pi} S(f)(\theta)^{p} w(\theta) d \theta\right)^{1 / p} \leq c\left(\int_{0}^{2 \pi} f^{*}(\theta)^{p} w(\theta) d \theta\right)^{1 / p}
$$

Moreover, if $F$ is an analytic function whose real part is $f$ then

$$
\left(\int_{0}^{2 \pi} S(F)^{p}(\theta) w(\theta) d \theta\right)^{1 / p} \leq c\left(\int_{0}^{2 \pi} f^{*}(\theta)^{p} w(\theta) d \theta\right)^{1 / p}
$$

This will complete the proof of Theorem 1 . For if $1<p<2$ and $w$ satisfies $A_{p}$ (which implies that $w$ satisfies $A_{2}$ ) then by Lemmas 4 and 2 ,

$$
\left(\int_{0}^{2 \pi} f^{*}(\theta)^{p} w(\theta) d \theta\right)^{1 / p} \leq c\left(\int_{0}^{2 \pi}|f(\theta)|^{p} w(\theta) d \theta\right)^{1 / p}
$$

It is not hard to prove Theorem 3 using Theorem 2. Since $S(F)(\theta)=$ $2 S(f)(\theta)$ for $F=f+i f$, it is enough to show the version for harmonic $f$. But

$$
\alpha^{-2} \int_{\left\{f^{*} \leq a\right\}} f^{* 2} w d \theta=-\alpha^{-2} \int_{0}^{a} t^{2} d \lambda(t) \leq \alpha^{-2} \int_{0}^{a} t \lambda(t) d t,
$$

where $\lambda(t)=\int_{\left\{f^{*}>t\right\}} w(\theta) d \theta$ is the distribution function of $f^{*}$ with respect to the measure $w(\theta) d \theta$. Thus by Theorem 2 ,

$$
\int_{\{S(f)>a\}} w d \theta \leq c\left[\lambda(\alpha)+a^{-2} \int_{0}^{a} t \lambda(t) d t\right] \text {. }
$$

Since the left side is the value at $a$ of the distribution function of $S(f)$ with respect to $w(\theta) d \theta$, we obtain that for $p>0$,

$$
\begin{array}{r}
\int_{0}^{2 \pi} S(f)^{p}(\theta) w(\theta) d \theta \leq p \int_{0}^{\infty} \alpha^{p-1}\left[\lambda(\alpha)+\alpha^{-2} \int_{0}^{a} t \lambda(t) d t\right] d \alpha \\
=p \int_{0}^{\infty} \alpha^{p-1} \lambda(\alpha) d \alpha+p \int_{0}^{\infty} t \lambda(t)\left[\int_{t}^{\infty} \alpha^{p-3} d \alpha\right] d t .
\end{array}
$$


If $p<2$, this is just a constant depending on $p$ times

$$
p \int_{0}^{\infty} t^{p-1} \lambda(t) d t=\int_{0}^{2 \pi} f^{*}(\theta)^{p} w(\theta) d \theta
$$

As a final corollary of Theorem 2 we also obtain the following weak-type result for $p=1$.

Theorem 4. Let $w$ be any weight which satisfies $A_{1}$. Suppose $f(\theta)$ satisfies $\int_{0}^{2 \pi}|f(\theta)| w(\theta) d \theta<\infty$, and let $f\left(r e^{i \theta}\right)$ denote the Poisson integral of $f$. Then for $a>0$,

$$
\int_{\{\theta: S(f)(\theta)>a\}} w(\theta) d \theta \leq \frac{c}{\alpha} \int_{0}^{2 \pi}|f(\theta)| w(\theta) d \theta
$$

with $c$ independent of $f$ and $a$.

To see this, we first observe that $w$ is bounded below by a positive constant. Indeed, by condition $A_{1}, c w(\phi) \geq(1 / 2 \pi) \int_{0}^{2 \pi} w(\theta) d \theta$ for all $\phi \in(0,2 \pi)$. From this it follows that $f$ is integrable. From Theorem 2,

$$
\int_{\{S(f)>\alpha\}} w(\theta) d \theta \leq c\left[\lambda(\alpha)+\alpha^{-2} \int_{0}^{a} t \lambda(t) d t\right]
$$

where $\lambda(t)=\int_{\left\{f^{*}>t\right\}} w(\theta) d \theta$. By [5 (cf. Lemmas 4 and 2)] condition $A_{1}$ implies that $t \lambda(t) \leq c \int_{0}^{2 \pi}|f(\theta)| w(\theta) d \theta$. Theorem 4 follows immediately.

We now turn to the proof of Theorem 2 , beginning with some additional notation. With $\alpha>0$ fixed, denote $B=\left\{f^{*}>\alpha\right\}$ and $E=\left\{f^{*} \leq \alpha\right\}$. Write the open set $B$ as the union $B=\bigcup_{i} B_{i}$ of open intervals $B_{i}$ on $|z|=1$. Then if $\Omega=$ $\bigcup_{\theta \in E} \Gamma(\theta), \Omega$ is the complement in $|z|<1$ of "triangular" regions with bases $B_{i}$. If $(\partial \Omega)^{1}$ is the part of $\partial \Omega$ in $|z|<1$, then $(\partial \Omega)^{1}$ is the union of the tops $C_{i}$ of the triangular regions with bases $B_{i}$.

From the fact that $\Gamma \subset \Gamma_{1}$ it follows that $|f| \leq \alpha$ in $\Omega$-moreover, each point of $C_{i}$ is the center of a circle in which $|f| \leq a$ whose radius is proportional to the distance from the point to $|z|=1$. From this and the mean-value property of harmonic functions, it follows easily that $\left(1-r^{2}\right)|\nabla f| \leq c \alpha$ on $(\partial \Omega)^{1}$. (See also the remark following Lemma 4.)

For convenience, we will now make several assumptions about $f$ and $w$. These will be dropped later. We will assume that $f$ is smooth up to and including $|z|=1$, and that it is the Poisson integral of its boundary values $f(\theta)$. Since $w$ is a Helson-Szegö weight, it can be written $w(\theta)=e^{u(\theta)+\widetilde{v}(\theta)}$ where $u$ is bounded and $|v| \leq c<\pi / 2$. We may clearly assume without loss of generality that $u=0$. Thus $w(\theta)=e^{\widetilde{v}(\theta)}$, and we will suppose that $v(\theta)$ and $\tilde{v}(\theta)$ are smooth functions on $(0,2 \pi)$. 
Since

$$
\int_{\{S(f)>a\}} w d \theta \leq \int_{\{S(f)>a\} \cap E} w d \theta+\int_{B} w d \theta
$$

it is enough to prove an estimate of the kind (4.3) for $\int_{\{S(f)>a\} \cap E} w d \theta$. By Tschebyshev's inequality,

$$
\int_{\{S(f)>a\} \cap E} w d \theta \leq a^{-2} \int_{E} S(f)^{2}(\theta) w(\theta) d \theta
$$

Substituting

$$
S(f)^{2}(\theta)=\iint_{\Gamma(\theta)}\left|\nabla f\left(r e^{i \phi}\right)\right|^{2} r d r d \phi
$$

and changing the order of integration, we obtain

$$
\int_{\{S(f)>\alpha\} \cap E} w d \theta \leq c \alpha^{-2} \iint_{\boldsymbol{\Omega}}\left(1-r^{2}\right) w\left(r e^{i \phi}\right)\left|\nabla f\left(r e^{i \phi}\right)\right|^{2} r d r d \phi .
$$

Here we have used the facts that $\Omega=\bigcup_{\theta \in E} \Gamma(\theta)$ and that $\int_{\gamma|\theta-\phi| 1-r} w d \theta$ $\leq c(1-r) w\left(r e^{i \phi}\right)$ (cf. also Lemma 1).

By (2.3),

$$
\begin{aligned}
c \alpha^{2} \int_{\{S(f)>a\} \cap E} w d \theta & \leq \iint_{\Omega}\left(1-r^{2}\right)\left|W\left(r e^{i \phi}\right)\right|\left|\nabla f\left(r e^{i \phi}\right)\right|^{2} r d r d \phi \\
& =\iint_{\Omega}\left(1-r^{2}\right) e^{\widetilde{v}\left(r e^{i \phi}\right)} \Delta f^{2}\left(r e^{i \phi}\right) r d r d \phi .
\end{aligned}
$$

Since $\Delta\left(e^{\tilde{v}} f^{2}\right)=f^{2} \Delta e^{\tilde{v}}+e^{\tilde{v}} \Delta\left(f^{2}\right)+2 \nabla e^{\tilde{v}} \cdot \nabla\left(f^{2}\right)$, the last expression (4.4) equals

$$
\begin{aligned}
\iint_{\mathbf{\Omega}}\left(1-r^{2}\right) \Delta\left(e^{\widetilde{v}} f^{2}\right) r d r d \phi & -\iint_{\Omega}\left(1-r^{2}\right) f^{2} \Delta e^{\widetilde{v}} r d r d \phi \\
& -2 \iint_{\Omega}\left(1-r^{2}\right) \nabla e^{\widetilde{v}} \cdot \nabla \cdot\left(f^{2}\right) r d r d \phi=I+I I+I I I .
\end{aligned}
$$

Here $I I$ is negative, so we simply drop it. (We could estimate II directly and in fact will essentially do this when we estimate III.) For $I$, we use Green's theorem( $\left.{ }^{2}\right)$, observe that $\Delta\left(1-r^{2}\right)=-4<0$ and obtain

$$
\begin{aligned}
I & \leq \int_{\partial \Omega}\left[\left(1-r^{2}\right)\left|\frac{\partial}{\partial \eta}\left(e^{\tilde{v}} f^{2}\right)\right|+\left|\frac{\partial}{\partial \eta}\left(1-r^{2}\right)\right| e^{\tilde{v}} f^{2}\right] d \sigma \\
& \leq c \int_{\partial \Omega}\left[\left(1-r^{2}\right) e^{\tilde{v}}|f||\nabla f|+\left(1-r^{2}\right) e^{\tilde{v}}|\nabla v| f^{2}+e^{\widetilde{v}} f^{2}\right] d \sigma,
\end{aligned}
$$

since $|\nabla \tilde{v}|=|\nabla v|$ by the Cauchy-Riemann equations. Write the last integral as the sum of two, one extended over $E=\partial \Omega \cap\{|z|=1\}$ and the other over $(\partial \Omega)^{1}$.

(2) Here we meet the usual technical problems caused by the fact that $\partial \Omega$ is not smooth. These can easily be overcome by using appropriate approximating domains and passing to the limit, keeping in mind that all functions are smooth up to $|z|=1$. The method is standard, and we will not give the details. 
Since all functions are smooth on $|z| \leq 1$ and $r=1$ on $E$, the part over $E$ is at most

$$
\int_{E} f^{*}(\theta)^{2} e^{\widetilde{v}(\theta)} d \theta=\int_{E} f^{* 2}(\theta) w(\theta) d \theta
$$

For the part over $(\partial \Omega)^{1}$, we recall that $|f| \leq \alpha$ on $(\partial \Omega)^{1}$. Moreover, $\left(1-r^{2}\right)|\nabla f| \leq c \alpha$ on $(\partial \Omega)^{1}$, and since $v$ is bounded, $\left(1-r^{2}\right)|\nabla v| \leq c$. Thus the part extended over $(\partial \Omega)^{1}$ is at most a constant times

$$
\alpha^{2} \int_{(\partial \Omega)^{1}} e^{\widetilde{\nu}\left(r e^{i \phi}\right)} d \sigma
$$

By (2.3),

$$
\alpha^{2} \int_{(\partial \boldsymbol{\Omega})^{1}} e^{\widetilde{\nu}\left(r e^{i \theta}\right)} d \sigma \leq c \alpha^{2} \int_{(\partial \Omega)^{l}} w\left(r e^{i \theta}\right) d \sigma=c a^{2} \sum_{j} \int_{C_{j}} w\left(r e^{i \theta}\right) d \sigma .
$$

The lemma which follows shows that there exists a constant $c$ independent of $j$ such that $\int_{C_{j}} w\left(r e^{i \theta}\right) d \sigma \leq c \int_{B_{j}} w(\theta) d \theta$. Adding over $j$ and recalling that $B$ is the disjoint union of the $B_{j}$, we obtain the required estimate

$$
I \leq c\left[\int_{E} f^{*}(\theta)^{2} w(\theta) d \theta+\alpha^{2} \int_{B} w(\theta) d \theta\right] .
$$

Lemma 7. Let $B$ be an interval on $|z|=1$ and let $C$ denote the two line segments which form the top of the standard "triangular" region with base $B$. Let $I$ be an interval lying along one side of $C$ whose length is at least proportional to its distance from $|z|=1$; that is, if $s e^{i \phi_{1}}$ is the center of $I$, then $|I|$ $\geq c(1-s)$ for some constant $c .(3)$ Let $(a, b)$ denote the projection of I onto $|z|=1$. Then for any $w$ satisfying (2.5) and condition $A_{p}$ for some $p, 1<p<\infty$,

$$
\int_{I} w\left(r e^{i \theta}\right) d \sigma \leq c \int_{a}^{b} w(\theta) d \theta .
$$

Proof. We may assume without loss of generality that $B=(0, \psi)$ and $(a, b)$ $C(0, \psi / 2)$. By $(2.5)$

$$
w\left(r e^{i \phi}\right) \approx \frac{1}{1-r} \int_{|\theta-\phi|<1-r} w(\phi) d \phi .
$$

For $r e^{i \theta} \in I, 1-r \approx \theta$ so that by the equivalence above and Harnack's principle,

$$
\begin{aligned}
\int_{I} w\left(r e^{i \theta}\right) d \sigma & \approx \int_{a}^{b}\left\{\frac{1}{\theta} \int_{0}^{2 \theta} w(\phi) d \phi\right\} d \theta \\
& \leq \int_{0}^{2 b} w(\phi)\left\{\int_{\phi / 2}^{b} \frac{d \theta}{\theta}\right\} d \phi=\int_{0}^{2 b} w(\phi) \log \frac{2 b}{\phi} d \phi .
\end{aligned}
$$

Applying Hölder's inequality with exponents $p_{1}>1$ and $p_{1}^{\prime}$, we obtain

$$
\int_{I} w\left(r e^{i \phi}\right) d \sigma \leq c\left(\int_{0}^{2 b} w(\phi)^{p} 1 \phi\right)^{1 / p} 1\left(\int_{0}^{2 b}\left[\log \frac{2 b}{\phi}\right]^{p} d \phi\right)^{1 / p_{1}^{\prime}} .
$$

(3) Here $c$ denotes positive constants independent of $I$ and $B$. 
Here

$$
\int_{0}^{2 b}\left[\log \frac{2 b}{\phi}\right]^{p_{1}^{\prime}} d \phi=b \int_{0}^{2}\left[\log \frac{2}{\phi}\right]^{p_{1}^{\prime}} d \phi=c_{p_{1}} b
$$

Thus

$$
\int_{I} w\left(r e^{i \phi}\right) d \sigma \leq c_{p_{1}} b\left(\frac{1}{b} \int_{0}^{2 b} w(\phi)^{p_{1}} d \phi\right)^{1 / p_{1}} \leq c_{p} \int_{0}^{2 b} w(\phi) d \phi
$$

by Lemma 3 , choosing $p_{1}$ sufficiently close to 1 . The interval $(0,2 b)$ of integration contains $(a, b)$. Moreover, since the length of $I$ exceeds a constant times its distance from $|z|=1$, the length of $(a, b)$ exceeds a constant times that of $(0,2 b)$. But then it follows immediately from (2.5) and Harnack's principle that $\int_{0}^{2 b} w(\phi) d \phi \approx \int_{a}^{b} w(\phi) d \phi$, completing the proof.

It remains to estimate $I I I$, which is somewhat more involved. Since $\left|\nabla e^{\tilde{\nu}}\right|=$ $e^{\tilde{v}}|\nabla \tilde{v}|$

$$
I I I \leq \iint_{\Omega}\left(1-r^{2}\right) e^{\tilde{v}}|\nabla \widetilde{v}||f||\nabla f| r d r d \phi,
$$

which by Schwarz's inequality is at most

$$
\left(\left.\int_{\Omega} \int_{\Omega}\left(1-r^{2}\right) e^{\widetilde{v}} \nabla \widetilde{v}\right|^{2} f^{2} r d r d \phi\right)^{1 / 2}\left(\iint_{\Omega}\left(1-r^{2}\right) e^{\widetilde{v}}|\nabla f|^{2} r d r d \phi\right)^{1 / 2}
$$

Denote

$$
X=\iint_{\mathbf{\Omega}}\left(1-r^{2}\right) e^{\tilde{v}} \Delta\left(f^{2}\right) r d r d \phi, \quad Y=\int_{E} f^{* 2} w d \theta+\alpha^{2} \int_{B} w d \theta
$$

and

$$
Z=\iint_{\mathbf{\Omega}}\left(1-r^{2}\right) e^{\tilde{v}}|\Delta \tilde{v}|^{2} f^{2} r d r d \phi
$$

Since $|\nabla f|^{2}=1 / 2 \Delta\left(f^{2}\right)$, we obtain from (4.4), (4.5) and the estimate above for III that $X \leq c\left(Y+X^{1 / 2} Z^{1 / 2}\right)$. If we show that $Z \leq c Y$ we will have $X \leq c\left(Y+X^{1 / 2} Y^{1 / 2}\right)$ and therefore $X \leq c Y$, which.will establish Theorem 2. The rest of this section is devoted to proving that $Z \leq c Y$.

Write

$$
Z=\iint_{\Omega} f^{2} d \mu
$$

where, since $\Delta e^{\tilde{v}}=e^{\tilde{\nu}}|\nabla \tilde{v}|^{2}$,

$$
d \mu\left(r e^{i \phi}\right)=\left(1-r^{2}\right) \Delta e^{\widetilde{v}\left(r e^{i \phi}\right)} r d r d \phi .
$$

The idea of the proof is to find an analogue of Lemma 5 for $\Omega$. One complication is that $Y$ is an integration over $|z|=1$ with respect to Lebesgue measure, rather than one over $\partial \Omega$ with respect to harmonic measure. 
As always we identify $\phi=e^{i \phi}$, and let $\phi^{\prime}=r e^{i \phi}$ denote the point on $\partial \Omega$ whose projection onto $|z|=1$ is $\phi$. Let

$$
\bar{f}(\phi)= \begin{cases}f(\phi), & \phi \in E, \\ f\left(\phi^{\prime}\right), & \phi \in B .\end{cases}
$$

Thus $\bar{f}$ is a continuous function defined on $|z|=1$. Let $\bar{f}\left(r e^{i \phi}\right)$ denote the Poisson integral of $\bar{f}$ and let

$$
\bar{f}^{*}(\phi)=\sup _{\Gamma_{2}(\phi)} \bar{f}\left(r e^{i \theta}\right)
$$

where $\Gamma_{2} \subset \Gamma$. We claim there exist constants $c$ and $c_{1}$ such that for every $\beta>0$,

$$
\mu\left\{r e^{i \phi} \in \Omega:\left|f\left(r e^{i \phi}\right)\right|>\beta\right\} \leq c \int_{\left\{F^{*}>c_{1} \beta\right\}} w(\phi) d \phi .
$$

Taking this inequality for distribution functions temporarily for granted, let us show that it implies $Z \leq c Y$. In fact, we obtain immediately from (4.8) that

$$
\iint_{\Omega}|f|^{p} d \mu \leq c \int_{0}^{2 \pi}\left\{\bar{f}^{*}\right\}^{p} w d \phi, \quad p>0 .
$$

Taking $p=2$ and recalling that $w$ satisfies $A_{2}$, we get from Lemma 2 that

$$
\begin{aligned}
Z & =\iint_{\Omega} f^{2} d \mu \leq c \int_{0}^{2 \pi} \bar{f}^{2}(\phi) w(\phi) d \phi \\
& =c\left[\int_{E} f^{2}(\phi) w(\phi) d \phi+\int_{B} f^{2}\left(\phi^{\prime}\right) w(\phi) d \phi\right] \\
& \leq c\left[\int_{E} f^{* 2} w d \phi+\alpha^{2} \int_{B} w d \phi\right]=c Y .
\end{aligned}
$$

We will prove (4.8) in two steps. We assume without loss of generality that $f \geq 0$ and define for $\phi^{\prime} \in \partial \Omega$ the function

$$
f^{* *}\left(\phi^{\prime}\right)=\operatorname{Sup}_{\Gamma_{2}\left(\phi^{\prime}\right)} f\left(r e^{i \theta}\right) .
$$

Thus $f^{* *}$ is defined on $\partial \Omega$. Let $G^{\prime}=\left\{\phi^{\prime} \in \partial \Omega: f^{* *}\left(\phi^{\prime}\right)>\beta\right\}$ so that $G^{\prime}$ is the disjoint union of "intervals" $I_{i}$ along $\partial \Omega$. Then $\left\{r e^{i \phi} \in \Omega: f\left(r e^{i \phi}\right)>\beta\right\} \subset$ $\bigcup_{i} T_{i}$, where $T_{i}$ are the "triangular" regions with bases $I_{i}$ which form the complement in $\Omega$ of $\bigcup_{\phi^{\prime} \notin G^{\prime}} \Gamma_{2}\left(\phi^{\prime}\right)$. Thus

$$
\mu\left\{r e^{i \phi} \in \Omega: f\left(r e^{i \phi}\right)>\beta\right\} \leq \sum \mu\left(T_{i}\right),
$$

and our first step in proving (4.8) will be showing that 


$$
\sum_{i} \mu\left(T_{i}\right) \leq c \int_{G} w(\phi) d \phi
$$

where $G \subset\{|z|=1\}$ is the projection onto $|z|=1$ of $\left\{\phi^{\prime} \in \partial \Omega: f^{* *}\left(\phi^{\prime}\right)>c_{1} \beta\right\}$, $0<c_{1}<1$.

Now $\mu\left(T_{i}\right)=\iint_{T_{i}}\left(1-r^{2}\right) \Delta e^{\tilde{v}} r d r d \phi$. If we apply Green's theorem as in $\S 3$ and observe that $\Delta\left(1-r^{2}\right)=-4<0$, then

$$
\mu\left(T_{i}\right) \leq \int_{\partial T_{i}}\left[\left|\frac{\partial}{\partial \eta}\left(1-r^{2}\right)\right| e^{\tilde{\nu}}+\left(1-r^{2}\right)\left|\frac{\partial}{\partial \eta} e^{\tilde{v}}\right|\right] d \sigma .
$$

Since $e^{\tilde{v}} \leq c w,\left|\partial\left(1-r^{2}\right) / \partial \eta\right| e^{\tilde{v}} \leq c w$ and $\left(1-r^{2}\right)\left|\partial e^{\widetilde{v}} / \partial \eta\right| \leq c w\left(1-r^{2}\right)|\nabla \tilde{\nu}|=$ $c w\left(1-r^{2}\right)|\nabla v| \leq c w$. Thus

$$
\mu\left(T_{i}\right) \leq c \int_{\partial T_{i}} w\left(r e^{i \phi}\right) d \sigma
$$

For any $T_{i}$ whose base $I_{i}$ lies entirely on $|z|=1$ we have from (4.10) and Lemma 7 that

$$
\mu\left(T_{i}\right) \leq c \int_{l_{i}} w(\phi) d \phi .
$$

Adding over $i$ and noting that the union of such $I_{i}$ is a subset of both $|z|=1$ and $G^{\prime}$, and therefore, is a subset of $G$, we obtain (4.9) for such $T_{i}$.

Consider next any $T_{i}$ whose base $I_{i}$ lies entirely on one side of the top $C_{j}$ of the triangular region whose base $B_{j}$ is an interval of $B$. If the length of $I_{i}$ exceeds a fixed constant multiple of its distance to $|z|=1$, we can again use Lemma 7 (for each side of $T_{i}$ ) and argue as above. Let us then suppose, on the other hand, that the length of $I_{i}$ is small compared to its distance from $|z|=1$. Letting $\phi_{i}^{\prime}$ denote the center of $I_{i}$, we have by the definition of $G^{\prime}$ that $f\left(z_{i}\right)>\beta$ for some point $z_{i} \in \Gamma_{2}\left(\phi_{i}^{\prime}\right)$. Since $f$ is positive and harmonic in $|z|<1$, there exists by Harnack's principle a positive constant $c_{1}$ independent of $i$ such that $f(z)>c_{1} \beta$ for all $z$ in a circle with center $z_{i}$ and radius $c_{1}$ times the distance from $z_{i}$ to $|z|=1$. From this it follows immediately that $f^{* *}\left(\phi^{\prime}\right)>c_{1} \beta$ for an interval $I_{i}^{\prime}$ of points $\phi^{\prime}$ on $C_{j}$ containing $I_{i}$ which has the property of Lemma 7 . We now replace all those $I_{k}$ in $I_{i}^{\prime}$ by $I_{i}^{\prime}$ itself, and all the corresponding $T_{k}$ by the similar triangle with base $I_{i}^{\prime}$. Applying Lemma 7 and noting that the projection of $I_{i}^{\prime}$ onto $|z|=1$ lies in $G$, we obtain (4.9) in this case.

The remaining cases of (1) those $T_{i}$ whose bases contain points from both sides of a top $C_{j}$, and (2) those $T_{i}$ whose bases contain points of both the tops 
$C_{j}$ and the circle $|z|=1$ can be dealt with by combining the arguments above. This completes the proof of (4.9).

Our final step in proving (4.8) will be showing that $G$ lies in $\left\{\bar{f}^{*}>c \beta\right\}$. When combined with (4.9), this will prove (4.8). Showing that $G \subset\left\{f^{*}>c \beta\right\}$ is of course the same as showing that

$$
f^{* *}\left(\phi^{\prime}\right) \leq c \bar{f}^{*}(\phi)
$$

Since $f^{* *}\left(\phi^{\prime}\right)=\operatorname{Sup}_{\Gamma_{2}\left(\phi^{\prime}\right)} f\left(r e^{i \theta}\right)$ and $\bar{f}^{*}(\phi)=\operatorname{Sup}_{\Gamma_{2}(\phi)} \bar{f}\left(r e^{i \theta}\right)$, it follows from Lemma 4 that

$$
f^{* *}\left(\phi^{\prime}\right) \leq c \quad \operatorname{Sup}_{\gamma\left|\phi-\phi^{\prime}\right| \leq \epsilon \leq \pi} \epsilon^{-1} \int_{|\theta|<\epsilon} f(\phi+\theta) d \theta
$$

and

$$
\bar{f}^{*}(\phi) \geq c \operatorname{Sup}_{0<\epsilon \leq \pi} \epsilon^{-1} \int_{|\theta|<\epsilon} \bar{f}(\phi+\theta) d \theta .
$$

Suppose that $\phi \in B$, so that $\phi^{\prime}$ lies on a top $C_{j}$. Then by (4.12), the only values of $\epsilon$ required to determine $f^{* *}\left(\phi^{\prime}\right)$ are those values $\epsilon \geq \gamma\left|\phi-\phi^{\prime}\right|$, and therefore the integration in (4.12) is always extended over an interval around $\phi$ whose length is at least proportional to the distance from $\phi$ to $E$. We now need the following simple lemma.

Lemma 8. Let $B_{j}=\left(u_{j}, v_{j}\right)$ be an interval of $B$ and let $(a, b)$ be a subinterval of $B_{j}$ whose length exceeds a constant times its distance to the complement of $B_{j}$, i.e. $b-a \geq c_{1} \min \left\{a-u_{j}, v_{j}-b\right\}$. Then

$$
\int_{a}^{b} f(\theta) d \theta \leq c \int_{a}^{b} \bar{f}(\theta) d \theta
$$

with $c$ independent of $(a, b)$ and 7 .

Proof. By definition of $\bar{f}, \int_{a}^{b} \bar{f}(\theta) d \theta=\int_{a}^{b} f\left(\theta^{\prime}\right) d \theta$. For the proof we will assume $a-u_{j} \leq v_{j}-b$. For convenience, take $\left(u_{j}, v_{j}\right)=(0,2 A)$. Then $a<A$.

Case 1. $b \leq A$. Then the complex variable $\theta^{\prime}$, whose argument is $\theta$, has distance from $|z|=1$ equivalent to $\theta$. Thus by simple estimates on the Poisson kernel ([14, Vol. I, p. 96], and $|\sin u| \leq c|u|)$

$$
f\left(\theta^{\prime}\right) \geq c \int_{-\pi}^{\pi} f(y) \frac{\theta}{\theta^{2}+(\theta-y)^{2}} d y \geq c \int_{a}^{b} f(y) \frac{\theta}{\theta^{2}+(\theta-y)^{2}} d y .
$$

Therefore 


$$
\begin{aligned}
\int_{a}^{b} \bar{f}(\theta) d \theta & \geq c \int_{a}^{b} f(y)\left\{\int_{a}^{b} \frac{\theta d \theta}{\theta^{2}+(\theta-y)^{2}}\right\} d y \\
& \geq c \int_{a}^{b} f(y)\left\{\frac{1}{b^{2}} \int_{a}^{b} \theta d \theta\right\} d y \geq c \int_{a}^{b} f(y) d y,
\end{aligned}
$$

since by hypothesis there exists a constant $c_{1}$ satisfying $0<c_{1}<1$ and $c_{1} b$ $>a$.

Case 2. $b>A$. If for a fixed $c, 1<c<2, b>c A$ then since $a-u_{j} \leq v_{j}-b$ both subintervals $(a, A)$ and $(A, b)$ of $(a, b)$ are of the type considered in Case 1. The result follows by adding. If $b \leq c A$, then since $f$ is nonnegative we have by Harnack's principle that $f\left(\theta^{\prime}\right)$ for $A \leq \theta \leq b$ is proportional to the value of $f$ at the point whose argument is $\theta$ and whose distance from $|z|=1$ is $\theta$. Thus we have, for all $a \leq \theta \leq b$,

$$
f\left(\theta^{\prime}\right) \geq c \int_{-\pi}^{\pi} f(y) \frac{\theta}{\theta^{2}+(\theta-y)^{2}} d y,
$$

and we may continue the argument as in Case 1. This completes the proof of Lemma 8.

The claim (4.11) follows easily from Lemma 8 and the expressions above for $f^{* *}$ and $\bar{f}^{*}$. In fact, if $\phi \in B$ we have already noted that the integration in (4.12) is always over an interval whose length is at least proportional to the distance from $\phi$ to $E$. Thus for $\epsilon \geq \gamma\left|\phi-\phi^{\prime}\right|$,

$$
\int_{|\theta|<\epsilon} f(\phi+\theta) d \theta=\int_{R_{1}}+\int_{R_{2}}+\int_{R_{3}}
$$

where $R_{1}$ is the part of the interval of $B$ containing $\phi$ in $(\phi-\epsilon, \phi+\epsilon), R_{2}$ is the rest of $(\phi-\epsilon, \phi+\epsilon)$ in $B$, and $R_{3}$ is the part of $(\phi-\epsilon, \phi+\epsilon)$ in $E$. Since $R_{1}$ is the type of interval $(a, b)$ of Lemma 8, we have $\int_{R_{1}} f d \theta \leq c \int_{R_{1}} \bar{f} d \theta$. Since $(\phi-\epsilon, \phi+\epsilon)$ is a whole interval, Lemma 8 also implies the same inequality with $R_{1}$ replaced by $R_{2}$. Finally, since $f=\bar{f}$ in $E, \int_{R_{3}} f d \theta=\int_{R_{3}} \bar{f} d \theta$. Thus

$$
\int_{|\theta|<\epsilon} f(\phi+\theta) d \theta \leq c \int_{|\theta|<\epsilon} \bar{f}(\phi+\theta) d \theta
$$

for such $\epsilon$, and (4.11) follows.

In case $\phi \in E$, we must consider $\left.\int|\theta|<\epsilon\right](\phi+\theta) d \theta$ for all $\epsilon>0$. In this case, however, the integral splits into parts of kind $R_{2}$ and $R_{3}$ only, and (4.11) follows as above.

The proof of Theorem 2 is now complete in the case that $f, v$ and $\tilde{v}$ are smooth in $|z| \leq 1$. It is not difficult to remove these assumptions by approximation 
arguments. Let $v(\theta)$ be any function satisfying $|v(\theta)| \leq c<\pi / 2$ and let $w(\theta)=$ $e^{\widetilde{\nu}(\theta)}$. Then $\left|v\left(\rho e^{i \theta}\right)\right| \leq c<\pi / 2$, and as functions of $\theta$ for fixed $\rho<1$, both $v\left(\rho e^{i \theta}\right)$ and its conjugate $\tilde{v}\left(\rho e^{i \theta}\right)$ have Poisson integrals which are smooth in $|z| \leq 1$. Thus $w_{\rho}(\theta)=e^{\widetilde{\nu}\left(\rho_{e}{ }^{i \theta}\right)}$ satisfies the conclusion (4.3) of Theorem 2 . (We are still assuming $f$ is smooth in $|z| \leq 1$.) But $w_{\rho}(\theta) \approx w\left(\rho e^{i \theta}\right)$ by $(2.3)$, and (4.3) for $w$ follows from the $L^{1}$-convergence of $u\left(\rho e^{i \theta}\right.$ to $w(\theta)$. Finally, we can remove the assumption that $f$ be smooth in $|z| \leq 1$ by using $f_{\rho}\left(r e^{i \theta}\right)=$ $f\left(\rho r e^{i \theta}\right)$. For (4.3) holds for $f_{\rho}$, and $f_{\rho}^{*} / f^{*}$ and $S\left(f_{\rho}\right) / S(f)$ as $p / 1$. Thus (4.3) for $f$ follows without difficulty from the monotone convergence theorem.

\section{REFERENCES}

1. D. L. Burkholder, R. F. Gundy and M. L. Silverstein, A maximal function characterization of the class $H^{p}$, Trans. Amer. Math. Soc. 157 (1971), 137-153. MR 43 \#527.

2. A. P. Calderón and A. Zygmund, On the existence of certain singular integrals, Acta. Math. 88 (1952), 85-139. MR 14, 637.

3. L. Carleson, Interpolations by bounded analytic functions and the corona problem, Ann. of Math. (2) 76 (1962), 547-559. MR 25 \#5186.

4. C. Fefferman and E. M. Steim, $H^{p}$ spaces of several variables, Acta Math. 129 (1972), 137-193.

5. C. Fefferman and E. M. Stein, Some maximal inequalities, Amer. J. Math. 93 (1971), $107-115$.

6. V. F. Gapoškin, A generalization of the theory of M. Riesz on conjugate functions, Mat. Sb. 46 (88) (1958), 359-372. (Russian) MR 20 \#6000.

7. H. Helson and G. Szegö, A problem in prediction theory, Ann. Mat. Pura Appl. (4) 51 (1960), 107-138. MR $22 \# 12343$.

8. I. I. Hirschman, The decomposition of Walsh and Fourier series, Mem. Amer. Math. Soc. No. 15 (1955), 65 pp. MR 17, 257.

9. L. Hörmander, $L^{p}$ estimates for (pluri-)subharmonic functions, Math. Scand. 20 (1967), 65-78. MR 38 \#2323.

10. P. Krée, Sur les multiplicateurs dans $\mathcal{F} L^{p}$ avec poids, Ann. Inst. Fourier (Grenoble) 16 (1966), fasc. 2, 91-121. MR 35 \#7080.

11. J. Marcinkiewicz and A. Zygmund, A theorem of Lusin, Duke Math. J. 4 (1938), 473-485.

12. B. Muckenhoupt, Weighted norm inequalities for the Hardy maximal function, Trans. Amer. Math. Soc. 165 (1972), 207-226.

13. R. A. Hunt, B. Muckenhoupt and R. L. Wheeden, Weighted norm inequalities for the conjugate function and Hilbert transform, Trans. Amer. Math. Soc. 176 (1973), 227-251.

14. A. Zygmund, Trigonometrical series, 2nd rev. ed., Cambridge Univ. Press, New York, 1959. MR 21 \#6498.

FACULTAD DE CIENCIAS EXACTAS Y NATURALES, UNIVERSIDAD DE BUENOS AIRES, BUENOS AIRES, ARGENTINA

DEP ARTMENT OF MATHEMATICS, RUTGERS UNIVERSITY, NEW BRUNSWICK, NEW JERSEY 08903 